TRANSACTIONS OF THE

AMERICAN MATHEMATICAL SOCIETY

Volume 357, Number 4, Pages 1303-1324

S 0002-9947(04)03719-5

Article electronically published on November 4, 2004

\title{
AN UNUSUAL SELF-ADJOINT LINEAR PARTIAL DIFFERENTIAL OPERATOR
}

\author{
W. N. EVERITT, L. MARKUS, AND M. PLUM \\ Dedicated to Professor Johann Schröder
}

\begin{abstract}
In an American Mathematical Society Memoir, published in 2003, the authors Everitt and Markus apply their prior theory of symplectic algebra to the study of symmetric linear partial differential expressions, and the generation of self-adjoint differential operators in Sobolev Hilbert spaces. In the case when the differential expression has smooth coefficients on the closure of a bounded open region, in Euclidean space, and when the region has a smooth boundary, this theory leads to the construction of certain self-adjoint partial differential operators which cannot be defined by applying classical or generalized conditions on the boundary of the open region.

This present paper concerns the spectral properties of one of these unusual self-adjoint operators, sometimes called the "Harmonic" operator.

The boundary value problems considered in the Memoir (see above) and in this paper are called regular in that the cofficients of the differential expression do not have singularities within or on the boundary of the region; also the region is bounded and has a smooth boundary. Under these and some additional technical conditions it is shown in the Memoir, and emphasized in this present paper, that all the self-adjoint operators considered are explicitly determined on their domains by the partial differential expression; this property makes a remarkable comparison with the case of symmetric ordinary differential expressions.

In the regular ordinary case the spectrum of all the self-adjoint operators is discrete in that it consists of a countable number of eigenvalues with no finite point of accumulation, and each eigenvalue is of finite multiplicity. Thus the essential spectrum of all these operators is empty.

This spectral property extends to the present partial differential case for the classical Dirichlet and Neumann operators but not to the Harmonic operator. It is shown in this paper that the Harmonic operator has an eigenvalue of infinite multiplicity at the origin of the complex spectral plane; thus the essential spectrum of this operator is not empty.

Both the weak and strong formulations of the Harmonic boundary value problem are considered; these two formulations are shown to be equivalent.

In the final section of the paper examples are considered which show that the Harmonic operator, defined by the methods of symplectic algebra, has a domain that cannot be determined by applying either classical or generalized local conditions on the boundary of the region.
\end{abstract}

Received by the editors April 15, 2003.

2000 Mathematics Subject Classification. Primary 35J40, 35J67, 35P05; Secondary 32A36, $32 \mathrm{~A} 40,47 \mathrm{~B} 25$.

Key words and phrases. Linear partial differential equations, self-adjoint partial differential operators, spectral theory.

(C)2004 American Mathematical Society Reverts to public domain 28 years from publication 


\section{INTRODUCTION}

In the Memoir [8] there is exposed and developed a new general theory of boundary value problems for linear elliptic partial differential operators generated by elliptic differential expressions, of order $2 m(m \geq 1)$, in the form

$$
A(\mathbf{x}, D):=\sum_{0 \leq|s| \leq 2 m} a_{s}(\mathbf{x}) D^{s} \text { for all } \mathbf{x} \in \Omega,
$$

with complex-valued coefficients $a_{s}(\cdot) \in C^{\infty}(\bar{\Omega})$ for $0 \leq|s| \leq 2 m$, defined on a bounded region $\Omega$ (open connected set) with $C^{\infty}$-smooth boundary $\partial \Omega$ in the real Euclidean space $\mathbb{E}^{r}(r \geq 2)$, and for the corresponding linear operators in the Hilbert space $L_{2}(\Omega)$.

In particular, consider such a linear operator $A$ defined by

$$
A: f \rightarrow A(\cdot, D) f=\sum_{0 \leq|s| \leq 2 m} a_{s}(\cdot) D^{s} f \text { for all } f \in D(A)
$$

with

$$
A(\mathbf{x}, D) f=\sum_{0 \leq|s| \leq 2 m} a_{s}(\mathbf{x})\left(D^{s} f\right)(\mathbf{x}) \text { for all } \mathbf{x} \in \Omega
$$

on the classical domain

$$
D(A):=C_{0}^{\infty}(\Omega) .
$$

We use the symbol $A(\cdot, D)$ for the differential expression given by (1.1) which can be applied using classical derivatives to functions in $C^{2 m}(\Omega)$ (see (1.13)) and using weak derivatives to functions in the Sobolev Hilbert space $W^{2 m}(\Omega)$; see (1.9) and (1.10).

An extension of this operator $A$ to the Sobolev Hilbert space $W^{2 m}(\Omega)$ (see (1.5) and (1.6) for instance) is then obtained by using weak derivatives for $D^{s}$ as usual, and thence a further extension to the maximal operator $T_{1}$ on the domain ${ }_{W}^{A m}(\Omega)$; see (1.20) and (1.26) below. It is a remarkable fact, as demonstrated in [8, Theorem 3.3], that this maximal operator can be expressed in the form $T_{1}: f \rightarrow A(\cdot, D) f$, for $f \in \stackrel{A}{W}^{2 m}(\Omega)$, through this usage of weak derivatives. This result parallels the theory of ordinary classical and quasi-differential expressions (see [7]) and is a consequence of the assumptions, given above, that the boundary $\partial \Omega$ of the bounded region $\Omega$ and the coefficients of $A(\cdot, D)$ are all smooth. In some other cases of linear partial differential boundary value problems it is not possible to give such explicit information about the elements of the domains of the associated operators, or the role played by the differential expression.

The theory expounded in [8] effects the classification of all possible self-adjoint extensions $T$ of $A$ on domains $D(T) \subset L_{2}(\Omega)$ (for $A$ on $D(A)$ in (1.2) and (1.3)), and then treats the resulting spectral theory by new methods of complex symplectic algebra (see [7] and [8] for details), under the assumption of the following standing hypotheses:

Condition 1.1. We state

(a) $A(\cdot, D)$ is uniformly elliptic in the compact domain $\bar{\Omega}$, and the leading coefficients $\left\{a_{s}:|s|=2 m\right\}$ are real,

(b) $A(\cdot, D)$ is formally self-adjoint (Lagrange symmetric) so that the operator $A$ on $D(A)$, in (1.2) and (1.3), is symmetric in $L_{2}(\Omega)$, 
(c) the Dirichlet boundary value problem for $A(\cdot, D)$ in $\Omega$ is well-posed; that is,

$$
f \in W^{2 m}(\Omega) \cap \stackrel{\circ}{W^{m}}(\Omega) \text { and } A(\cdot, D) f=0 \text { imply } f=0 .
$$

Thus, under the hypotheses $(a)$ and $(b)$ of Condition 1.1 above, the linear map

$$
W^{2 m}(\Omega) \rightarrow L_{2}(\Omega) \times W^{2 m-1 / 2}(\partial \Omega) \times W^{2 m-3 / 2}(\partial \Omega) \times \cdots \times W^{m+1 / 2}(\partial \Omega)
$$

as given by (using the normal derivatives along the inwards unit normal $\mathbf{n}$ to $\partial \Omega$ )

$$
f \rightarrow\left\{A(\cdot, D) f,\left.f\right|_{\partial \Omega},\left.\frac{\partial f}{\partial \mathbf{n}}\right|_{\partial \Omega}, \cdots,\left.\frac{\partial^{m-1} f}{\partial \mathbf{n}^{m-1}}\right|_{\partial \Omega}\right\},
$$

is a bounded Fredholm map of index 0, a statement which requires in particular the trace theorem; see [1], 8 and [15. Since the kernel in the mapping (1.5) and (1.6) is zero, according to the hypothesis (c) of Condition 1.1 (see (1.4)) this mapping defines an injective surjection with an inverse which is bounded according to the open mapping theorem. Hence (1.6) yields a continuous bijection (see (1.23) below for the definition of the Dirichlet operator $T_{D i r}$ )

$$
T_{\text {Dir }}: W^{2 m}(\Omega) \cap \stackrel{\circ}{W} m(\Omega) \rightarrow L_{2}(\Omega)
$$

of the Sobolev Hilbert subspace

$$
W^{2 m}(\Omega) \cap \stackrel{\circ}{W} m(\Omega) \subset W^{2 m}(\Omega)
$$

onto $L_{2}(\Omega)$, with a bounded inverse map $T_{D i r}^{-1}$.

It is of considerable interest to note that new kinds of domain conditions of a global, rather than a local, nature are required for the complete results in [8], in defining self-adjoint operators $T$ on $D(T)$, as above. Moreover, under such domain conditions, functions $f \in D(T) \subset L_{2}(\Omega)$ may well need to be specified without regard to boundary conditions involving the actual (or pointwise) values of $f$ or its derivatives on $\partial \Omega$ - either in the classical, or weak, or any other customary sense or interpretation.

In this paper the authors investigate in greater detail the domain of one of these quite unusual and exotic self-adjoint operators, namely the Harmonic operator $T_{\text {Har }}$ on $D\left(T_{H a r}\right)$, as defined and explained later in this section. In particular, in Theorem 2.1 below, the spectrum of $T_{\text {Har }}$ is analysed in considerable detail.

In this connection the spectral analysis for $T_{H a r}$ is recast in Section 3 below as a new kind of eigenvalue problem, and is related to the earlier work on the buckling elastic plate; see [14, Chapter 9, Sections 62 to 65].

Following these general investigations, we conclude this paper with a number of examples for the classical Laplacian $A(\cdot, D)=-\Delta$ in an open disk in $\mathbb{E}^{2}$, and also in an open ball in $\mathbb{E}^{3}$. These explicit examples in Section 4 display unexpected and spectacular irregularities for the boundary behaviour of functions in $D\left(T_{\text {Har }}\right)$.

Remark 1.2. Regular differential boundary value problems.

With the above stated conditions on the smoothness of the coefficients of the differential expression (1.1), the boundedness of the region $\Omega$ and, in particular, the smoothness of the boundary $\partial \Omega$, together with Condition 1.1, it is reasonable to regard this partial differential system as regular. The use of this term makes a good comparison with ordinary classical and quasi-derivative differential systems (see [7]) when the interval of the real line $R$ is compact, and the coefficients of the ordinary differential expression are all integrable on this interval. 
In the case of these regular ordinary boundary value problems the spectrum of any generated self-adjoint operator is discrete, i.e. consists only of isolated eigenvalues each of finite multiplicity; thus the essential spectrum of the operator is empty.

One of the results of this paper is to show that in spite of the smoothness conditions on the partial differential system given above, which warrant the use of the term regular, this spectral property of the ordinary differential case does not extend to the partial differential case. We show below, in Theorem 2.1, that the self-adjoint operator $T_{H a r}$ of (1.27) and (1.28) has a non-empty essential spectrum.

Remark 1.3. Clarification of notation.

While the investigations of this paper are essentially self-contained and can be understood independently from the Memoir [8], it is useful to observe that we employ a familiar and customary notation for the functional analysis of elliptic partial differential operators - as listed and explained fully in [8, Parts I and II of Appendix A], and further in the treatise [15]. For instance, we here review informally that

$$
L_{2}(\Omega):=\left\{f: \Omega \rightarrow \mathbb{C}:\|f\|^{2}=\int_{\Omega}|f(\mathbf{x})|^{2} d \mathbf{x}<\infty\right\}
$$

is the complex Hilbert space consisting of (equivalence classes of) complex-valued square-integrable functions in the bounded region $\Omega$ (open connected set) in $\mathbb{E}^{r}$, where $\mathbb{C}$ denotes the complex number field, $\mathbf{x}=\left(x_{1}, x_{2}, \ldots, x_{r}\right)$ are the real cartesian coordinates and $d \mathbf{x}$ indicates the Lebesgue measure of $\mathbb{E}^{r}$. As usual, the scalar product and norm of the functions $f, g \in L_{2}(\Omega)$ are denoted by

$$
\langle f, g\rangle:=\int_{\Omega} f(\mathbf{x}) \bar{g}(\mathbf{x}) d \mathbf{x} \text { and }\|f\|^{2}:=\langle f, f\rangle .
$$

Further we use the Sobolev Hilbert spaces, $W^{l}(\Omega)$, for each positive integer $l \in \mathbb{N} \equiv$ $\{1,2,3, \ldots\}$,

$$
W^{l}(\Omega):=\left\{f \in L_{2}(\Omega): D^{s} f \in L_{2}(\Omega) \text { for all }|s| \leq l\right\},
$$

where $D^{s}:=D_{1}^{s_{1}} D_{2}^{s_{2}} \cdots D_{r}^{s_{r}}$ are the weak (or distributional) partial derivatives of multi-index $s=\left(s_{1}, s_{2}, \ldots, s_{r}\right)$ and of total order $|s|=s_{1}+s_{2}+\cdots+s_{r} \leq l$. Here the norm and scalar product, for $f, g \in W^{l}(\Omega)$, are given by

$$
\|f\|_{l}^{2}:=\langle f, f\rangle_{l} \text { where }\langle f, g\rangle_{l}:=\sum_{0 \leq|s| \leq l}\left\langle D^{s} f, D^{s} g\right\rangle
$$

(and conventionally we can set $W^{0}(\Omega)=L_{2}(\Omega)$ ). Also the intermediate Sobolev Hilbert spaces $W^{\lambda}(\Omega)$, defined for all real $\lambda \geq 0$, play a role (see [8. Part I of Appendix A]) in the trace theory (as function spaces on $\partial \Omega$ ). In particular, as submanifolds of $L_{2}(\Omega)$, the proper inclusions, for $0<\lambda_{1}<\lambda_{2}$, obtain

$$
C_{0}^{\infty}(\Omega) \subset W^{\lambda_{2}}(\Omega) \subset W^{\lambda_{1}}(\Omega) \subset L_{2}(\Omega),
$$

with $C_{0}^{\infty}(\Omega)$ dense in $L_{2}(\Omega)$ (and similarly for the corresponding boundary function spaces on $\partial \Omega$ within $L_{2}(\partial \Omega)$; see [8, Parts I and II of Appendix A] and [15]).

We also denote by $\stackrel{\circ}{W}^{l}(\Omega) \subset W^{l}(\Omega)$ the proper Hilbert subspace

$$
\stackrel{\circ}{W}^{l}(\Omega):=\left\{f \in W^{l}(\Omega):\left.f\right|_{\partial \Omega}=0,\left.\frac{\partial f}{\partial \mathbf{n}}\right|_{\partial \Omega}=0, \ldots,\left.\frac{\partial^{l-1} f}{\partial \mathbf{n}^{l-1}}\right|_{\partial \Omega}=0\right\}
$$


in terms of the normal derivatives along the inwards unit normal $\mathbf{n}$ to $\partial \Omega$, as defined by the trace-map.

Equally we could define (using $W^{l}(\Omega)$-closures)

$$
W^{l}(\Omega):={\overline{C^{\infty}(\bar{\Omega})}}^{W^{l}(\Omega)} \text { and } \stackrel{\circ}{W}^{l}(\Omega):={\overline{C_{0}^{\infty}(\Omega)}}^{W^{l}(\Omega)} .
$$

Here we have used the customary notations of $C^{0}(\Omega)$ for continuous complexvalued functions on $\Omega$, and for each positive integer $k$

$$
C^{k}(\Omega):=\left\{f: \Omega \rightarrow \mathbb{C}: f \text { and } \frac{\partial^{|s|} f}{\partial x_{1}^{s_{1}} \cdots \partial x_{r}^{s_{r}}} \in C^{0}(\Omega) \text { for all }|s| \leq k\right\},
$$

where in this definition classical partial derivatives are implied.

Further, as usual, we define

$$
\begin{gathered}
C^{\infty}(\Omega):=\bigcap_{k \geq 0} C^{k}(\Omega), \\
C^{\infty}(\bar{\Omega}):=\left\{\left.f\right|_{\Omega}: f \in C^{\infty}\left(\mathbb{E}^{r}\right)\right\}, \\
C_{0}^{\infty}(\Omega):=\left\{f \in C^{\infty}(\Omega): \operatorname{supp}(f) \text { is a compact subset of } \Omega\right\} .
\end{gathered}
$$

These statements conclude Remark 1.3 on clarification of notation.

Following the theory in [8], based on the Stone-von Neumann theory of symmetric operators in Hilbert space as given in [6], we consider any self-adjoint operator $T$ on $D(T) \subset L_{2}(\Omega)$, specifically an extension of the operator $A$ (see (1.2) and (1.3) as generated by the partial differential expression $A(\cdot, D)$ in $\Omega$ (see (1.1)) with the standing hypotheses $(a),(b),(c)$ of Condition 1.1 above. The operator $T$ then necessarily lies between the minimal closed symmetric operator $T_{0}$, where

$$
T_{0}: f \rightarrow A(\cdot, D) f=\sum_{0 \leq|s| \leq 2 m} a_{s}(\cdot) D^{s} f \text { on } D\left(T_{0}\right)=\stackrel{\circ}{W}{ }^{2 m}(\Omega) \subset L_{2}(\Omega)
$$

( $T$ is an extension of $T_{0}$ to $D(T) \supset D\left(T_{0}\right)$ ), and the maximal operator (compare (1.26) below)

$$
T_{1}: f \rightarrow A(\cdot, D) f \text { on } D\left(T_{1}\right) \subset L_{2}(\Omega)
$$

( $T$ is a restriction of $T_{1}$ to $D(T) \subset D\left(T_{1}\right)$ ), where $T_{1}$ is the $L_{2}(\Omega)$-adjoint $T_{0}^{*}$ of $T_{0}$.

In prior analysis [8, Section 3, Theorem 3.3], using the hypotheses $(a),(b),(c)$ of Condition 1.1 it is shown that we can write

$$
D\left(T_{1}\right)=\stackrel{A}{W}{ }^{2 m}(\Omega),
$$

where the linear manifold ${ }_{W}^{A m}(\Omega)$ in $L_{2}(\Omega)$ is determined by the direct sum

$$
\stackrel{A}{W^{2 m}}(\Omega):=W^{2 m}(\Omega) \cap \stackrel{\circ}{W} m(\Omega) \dot{+} \stackrel{A}{L}(\Omega) .
$$

Namely, $\stackrel{A}{W}^{2 m}(\Omega)$ can be expressed as the direct sum of the two submanifolds of $L_{2}(\Omega)$,

$$
W^{2 m}(\Omega) \cap \stackrel{\circ}{W} m(\Omega)=\left\{f \in W^{2 m}(\Omega):\left.f\right|_{\partial \Omega}=0,\left.\frac{\partial f}{\partial \mathbf{n}}\right|_{\partial \Omega}=0, \ldots,\left.\frac{\partial^{m-1} f}{\partial \mathbf{n}^{m-1}}\right|_{\partial \Omega}=0\right\}
$$

and

$$
\stackrel{A}{L}_{2}(\Omega):=\left\{f \in L_{2}(\Omega): f \in C^{\infty}(\Omega) \text { and } A(\cdot, D) f=0 \text { in } \Omega\right\} .
$$


Remark 1.4. Note that in this definition (1.22) the condition $f \in C^{\infty}(\Omega)$ can be omitted since it follows from the conditions $f \in L_{2}(\Omega)$, and $A(\cdot, D) f=0$ in $\Omega$ in the distributional sense; see [9, Lecture 5, Theorem 5.1] or [10, Part 1, Chapter 16, Theorem 16.2 and its Corollary].

Remark 1.5. The operator $T_{1}$.

A more familiar expression for $D\left(T_{1}\right)$, as given in (1.19), is

$$
D\left(T_{1}\right)=\left\{f \in L_{2}(\Omega): A(\cdot, D) f \in L_{2}(\Omega)\right\},
$$

where $A(\cdot, D) f$ is understood in the distribution sense; this approach is not used here but we comment that it is shown in $[8$, that

$$
\stackrel{A}{W^{2 m}}(\Omega)=\operatorname{span}\left\{W^{2 m}(\Omega), \stackrel{A}{L_{2}}(\Omega)\right\},
$$

since for each $f \in W^{2 m}(\Omega)$, by hypothesis $(c)$ of Condition 1.1, there exists a unique function $\hat{f} \in W^{2 m}(\Omega) \cap \stackrel{A}{L_{2}}(\Omega)$ such that $f-\hat{f} \in W^{2 m}(\Omega) \cap \stackrel{\circ}{W}{ }^{m}(\Omega)$.

The linear manifold $W^{2 m}(\Omega) \cap \stackrel{\circ}{W}$ m $(\Omega)$ is the familiar space of all functions $f \in W^{2 m}(\Omega)$ which satisfy the homogeneous Dirichlet conditions on $\partial \Omega$, but $L_{2}(\Omega)$ is relatively unexplored. However, we now remark that $\stackrel{A}{L}_{2}(\Omega)$ is a closed Hilbert subspace of $L_{2}(\Omega)$ (compare also [8, Lemma 3.1]); for suppose that $\left(f_{n}: n \in \mathbb{N}\right)$ is a sequence in $\stackrel{A}{L}_{2}(\Omega)$ which converges to some $f$ in $L_{2}(\Omega)$. Then, for any $\varphi \in C_{0}^{\infty}(\Omega)$

$$
\int_{\Omega} f_{n} A(\cdot, D) \varphi d \mathbf{x}=0 \text { for all } n \in \mathbb{N}
$$

and hence

$$
\int_{\Omega} f A(\cdot, D) \varphi d \mathbf{x}=0
$$

thus $f \in \stackrel{A}{L_{2}}(\Omega)$; note Remark 1.4 .

We recall that the familiar Dirichlet operator

$$
T_{D i r}: f \rightarrow A(\cdot, D) f \text { for } f \in D\left(T_{D i r}\right):=W^{2 m}(\Omega) \cap \stackrel{\circ}{W^{m}}(\Omega)
$$

is a self-adjoint extension of $T_{0}$ on $D\left(T_{0}\right)$ in (1.17), with a discrete spectrum for which each eigenvalue is of finite multiplicity, and the collection of all eigenfunctions leads to an orthonormal basis for the Hilbert space $L_{2}(\Omega)$.

While $T_{D i r}$ is the restriction of $T_{1}$ to the domain $D\left(T_{D i r}\right) \subset W^{2 m}(\Omega)$, it is shown below that the domain $D\left(T_{\text {Har }}\right)$ of the self-adjoint operator $T_{\text {Har }}$ does not lie within $W^{2 m}(\Omega)$, but only within $\stackrel{A}{W}^{2 m}(\Omega)$ of $(1.20)$. In order to formulate the definition of $T_{\text {Har }}$ in (1.27) and (1.28) below, we first note that each function $f \in \operatorname{span}\left\{W^{2 m}(\Omega), \stackrel{A}{L_{2}}(\Omega)\right\} \subset L_{2}(\Omega)$ has a unique decomposition (according to Remark 1.5 above)

$$
f=f_{D}+f_{A}
$$

with

$$
f_{D} \in W^{2 m}(\Omega) \cap \stackrel{\circ}{W}^{m}(\Omega) \text { and } f_{A} \in \stackrel{A}{L_{2}}(\Omega) .
$$


Then, as proved in [ $\underline{8}$, Theorem 3.3], $T_{1}=T_{0}^{*}$ can also be given by

$$
T_{1} f=A(\cdot, D) f_{D} \text { for all } f \in D\left(T_{1}\right)
$$

so that $\operatorname{Ker}\left(T_{1}\right)=\stackrel{A}{L_{2}}(\Omega)$. As a consequence of this formula for $T_{1}$ it follows that

$$
T_{1} f_{A}=0 \text { for all } f \in \stackrel{A}{W^{2 m}}(\Omega)
$$

and this result is consistent with the fact that $A(\mathbf{x}, D) f_{A}=0$ for all $\mathbf{x} \in \Omega$. Since

$$
A(\cdot, D) f=A(\cdot, D) f_{D} \text { for all } f \in \stackrel{A}{W^{2 m}}(\Omega),
$$

we can write

$$
T_{1} f=A(\cdot, D) f=\sum_{0 \leq|s| \leq 2 m} a_{s}(\cdot) D^{s} f \text { for all } f \in D\left(T_{1}\right)=W^{A m}(\Omega) .
$$

Now define the Harmonic operator $T_{H a r}$ as the restriction of $T_{1}$ to the domain

$$
D\left(T_{\text {Har }}\right):=\stackrel{\circ}{W}{ }^{2 m}(\Omega)+\stackrel{A}{L}(\Omega) .
$$

One of the purposes of this paper is to explore $\stackrel{A}{L}_{2}(\Omega)$ and to investigate its significance for the operator $T_{H a r}$, in particular for the spectrum $\sigma\left(T_{H a r}\right)$, taking into account (see [1.27) and [8, Definition 4.2])

$$
T_{\text {Har }}: f \rightarrow A(\cdot, D) f \text { for all } f \in D\left(T_{\text {Har }}\right)=\stackrel{\circ}{W^{2 m}}(\Omega) \dot{+} \stackrel{A}{L}_{2}(\Omega)
$$

and

$$
\operatorname{Ker}\left(T_{\text {Har }}\right)=\stackrel{A}{L_{2}}(\Omega) .
$$

As previously mentioned, $T_{\text {Har }}$ is a self-adjoint extension of $A$ as given by (1.2) and (1.3), assuming the validity of Condition 1.1. We illustrate some of its interesting properties by several special examples presented in Section 4 below, using the classical Laplacian with the conventional negative sign,

$$
A(\cdot, D)=-\Delta:=-\sum_{j=1}^{r} D_{j}^{2},
$$

where $D_{j}=\partial / \partial x_{j}$, in a bounded region $\Omega \subset \mathbb{E}^{r}$, with corresponding linear operator

$$
A: f \rightarrow-\Delta f
$$

on its classical domain

$$
D(A)=C_{0}^{\infty}(\Omega) \subset L_{2}(\Omega) .
$$

In this situation the domain of the minimal operator $T_{0}$ is (see (1.17) $)$

$$
D\left(T_{0}\right)=\stackrel{\circ}{W}{ }^{2}(\Omega)
$$

and the domain of the maximal operator $T_{1}=T_{0}^{*}$ is denoted by the special notation (emphasising the Laplacian $\Delta$; see (1.19) )

$$
D\left(T_{1}\right)=\stackrel{\Delta}{W}^{2}(\Omega):=W^{2}(\Omega) \cap \stackrel{\circ}{W}{ }^{1}(\Omega)+\stackrel{\Delta}{L}_{2}(\Omega),
$$

where

$$
\stackrel{\Delta}{L}_{2}(\Omega)=\left\{f \in L_{2}(\Omega): f \in C^{\infty}(\Omega) \text { and } \Delta f \equiv 0 \text { in } \Omega\right\}
$$


The fact that $\stackrel{\Delta}{L}_{2}(\Omega)$ consists of all harmonic functions in $L_{2}(\Omega)$ accounts for the name of the Harmonic operator, defined here by

$$
T_{\text {Har }}: f \rightarrow-\Delta f
$$

on its domain

$$
D\left(T_{\text {Har }}\right)=\stackrel{\mathrm{o}}{W}^{2}(\Omega)+\stackrel{\Delta}{L}_{2}(\Omega),
$$

noting that the indicated direct sum is within $L_{2}(\Omega)$. In our examples in Section 4 with $\Omega$ the open unit disk in $\mathbb{E}^{2}$ (or the open unit ball in $\mathbb{E}^{3}$ ), we demonstrate explicitly that

$$
\stackrel{\Delta}{W}^{2}(\Omega) \nsubseteq W^{2}(\Omega)
$$

and furthermore that $D\left(T_{\text {Har }}\right)$ cannot be characterized by conditions localized to the boundary $\partial \Omega$, even though such a boundary value characterization may seem, at first glance, to be analogous to a regular problem for ordinary differential operators, see Remark 1.2 above.

We close this introductory section with a preliminary theorem.

Theorem 1.6. Let $A(\cdot, D)$ be an elliptic partial differential expression defined on a bounded region $\Omega \subset \mathbb{E}^{r}$, with corresponding linear operator $A$ on $D(A)=C_{0}^{\infty}(\Omega)$, as in (1.1) to (1.3) and satisfying Condition 1.1; and let $T_{0}$ and $T_{1}$ be the minimal and maximal operators for $A$ as in (1.17) to (1.22). Then there exists an orthogonal direct sum decomposition of the Hilbert space $L_{2}(\Omega)$

$$
L_{2}(\Omega)=T_{0}\left(\stackrel{o}{W^{2 m}}(\Omega)\right) \stackrel{\perp}{\oplus} \stackrel{A}{L}_{2}(\Omega) .
$$

Further, these two closed Hilbert subspaces have dimension

$$
\operatorname{dim}\left(T_{0}\left(\stackrel{o}{W^{2 m}}(\Omega)\right)\right)=\operatorname{dim}(\stackrel{A}{L}(\Omega))=\aleph_{0} .
$$

Proof. A consequence of Weyl's Lemma (see [8, Lemma 3.1] and Remark 1.5) asserts that

$$
\stackrel{A}{L}(\Omega)=\left[T_{0}\left(\stackrel{\circ}{W}{ }^{2 m}(\Omega)\right)\right]^{\perp}=\left\{T_{0} g: g \in \stackrel{\circ}{W} 2 m(\Omega)\right\}^{\perp} .
$$

We next demonstrate that $T_{0}\left(\stackrel{\circ}{W}{ }^{2 m}(\Omega)\right)$ is a closed subspace of $L_{2}(\Omega)$, which implies the result (1.38).

Let $\left\{g_{n} \in \stackrel{\mathrm{o}}{W}^{2 m}(\Omega): n \in \mathbb{N}\right\}$ be a sequence such that $\left\{T_{0} g_{n}: n \in \mathbb{N}\right\}$ is a Cauchy sequence in $L_{2}(\Omega)$. Because the map

$$
T_{\text {Dir }}: W^{2 m}(\Omega) \cap \stackrel{\circ}{W} m(\Omega) \rightarrow L_{2}(\Omega)
$$

is a bounded bijection onto $L_{2}(\Omega)$ with a bounded inverse (see (1.7) above), the sequence $\left\{g_{n}: n \in \mathbb{N}\right\}$ is a Cauchy sequence in the Sobolev norm of $W^{2 m}(\Omega)$. Thus there exists some $g \in W^{2 m}(\Omega)$ such that

$$
\lim _{n \rightarrow \infty}\left\|g_{n}-g\right\|_{2 m}=0 .
$$


Since $\stackrel{\mathrm{o}}{W}^{2 m}(\Omega)$ is closed in the Sobolev space $W^{2 m}(\Omega), g \in \stackrel{\mathrm{o}}{W}^{2 m}(\Omega)$. Moreover the map $T_{D i r}$ in (1.41) is bounded, and $T_{0}$ is the restriction of $T_{D i r}$ to $\stackrel{\circ}{W}^{2 m}(\Omega)$, so by (1.42)

$$
\lim _{n \rightarrow \infty} T_{0} g_{n}=T_{0} g
$$

in $L_{2}(\Omega)$. Hence $T_{0}\left(\stackrel{\mathrm{o}}{W}{ }^{2 m}(\Omega)\right)$ is a closed subspace of the Hilbert space $L_{2}(\Omega)$.

In order to prove (1.39) we recall that $L_{2}(\Omega)$ is a separable Hilbert space, so that each of the two subspaces $T_{0}\left(\stackrel{\circ}{W}{ }^{2 m}(\Omega)\right)$ and $\stackrel{A}{L}{ }_{2}(\Omega)$ is either of finite or denumerable dimension. Since $C_{0}^{\infty}(\Omega) \subset \stackrel{o}{W}^{2 m}(\Omega)$ is not finite dimensional (for each $k \in \mathbb{N}$ there exist $k$ functions $\varphi_{1}, \varphi_{2}, \ldots, \varphi_{k} \in C_{0}^{\infty}(\Omega)$ with pairwise disjoint supports), we observe that $\stackrel{\mathrm{o}}{W}^{2 m}(\Omega)$ is not finite dimensional. Further, the map $T_{D i r}$ in (1.41) is an injection, so we conclude that $T_{0}\left(\stackrel{\circ}{W^{2 m}}(\Omega)\right)$ is not finite dimensional, and hence

$$
\operatorname{dim}\left(T_{0}\left(\stackrel{\mathrm{o}}{W}^{2 m}(\Omega)\right)\right)=\aleph_{0}
$$

A similar argument holds for $\stackrel{A}{L}_{2}(\Omega)$. Clearly $C^{\infty}(\partial \Omega)$ is not finite dimensional; for each $k \in \mathbb{N}$ there exist $k$ functions $\psi_{1}, \psi_{2}, \ldots, \psi_{k} \in C^{\infty}(\partial \Omega)$ which form a linearly independent set over $\mathbb{C}$. Now define the functions $\left\{u_{j} \in W^{2 m}(\Omega): j=1,2, \ldots, k\right\}$ by

$$
A(\cdot, D) u_{j}=0,\left.u_{j}\right|_{\partial \Omega}=\psi_{j},\left.\frac{\partial u_{j}}{\partial \mathbf{n}}\right|_{\partial \Omega}=0, \ldots,\left.\frac{\partial^{m-1} u_{j}}{\partial \mathbf{n}^{m-1}}\right|_{\partial \Omega}=0 .
$$

The linear independence of the set $\left\{\psi_{1}, \psi_{2}, \ldots, \psi_{k}\right\}$ implies that the set

$$
\left\{u_{1}, u_{2}, \ldots, u_{k}\right\}
$$

is linearly independent in $\stackrel{A}{L}{ }_{2}(\Omega)$, so

$$
\operatorname{dim}\left(\begin{array}{l}
A \\
L
\end{array}(\Omega)\right)=\aleph_{0}
$$

\section{The Spectrum of $T_{\text {Har }}$}

The purpose of this section is to examine the spectrum of the self-adjoint operator $T_{\text {Har }}$ defined in (1.27) and (1.28); the essential results are collected in Theorem 2.1 using the notation and conditions of Theorem [1.6 above.

Theorem 2.1. Let $A(\cdot, D)$ be an elliptic partial differential expression on a bounded region $\Omega \subset \mathbb{E}^{r}$, with the corresponding minimal operator $T_{0}$ as defined in (1.17) above; let the corresponding Harmonic operator $T_{H a r}$ on $D\left(T_{H a r}\right)$ be given as in (1.27) and (1.28). Then

(a) The spectrum $\sigma\left(T_{H a r}\right)$ of the operator $T_{H a r}$ in $L_{2}(\Omega)$ consists of:

(i) the eigenvalue $\lambda=0$ of countable multiplicity, and

(ii) a discrete countable set of real non-zero eigenvalues each of finite multiplicity.

(b) Let $\left(\lambda_{n}: n \in \mathbb{N}\right)$ denote the sequence of all non-zero eigenvalues with elements repeated according to multiplicity; then $\lim _{n \rightarrow \infty}\left|\lambda_{n}\right|=\infty$. 
(c) The eigenspace of the eigenvalue 0 is precisely the linear manifold $\stackrel{A}{L}{ }_{2}(\Omega) \subset$ $D\left(T_{\text {Har }}\right)$ as defined in 1.22.

$(d)$ The eigenvalue sequence $\left(\lambda_{n}: n \in \mathbb{N}\right)$ of $(b)$ coincides with the eigenvalue sequence of the following boundary value problem in $L_{2}(\Omega)$, with the same corresponding eigenvalue multiplicities (recall that $D\left(T_{0}\right)=\stackrel{o}{W}{ }^{2 m}(\Omega)$ )

$$
\left\langle T_{0} v, T_{0} \varphi\right\rangle=\lambda\left\langle v, T_{0} \varphi\right\rangle \text { for all } \varphi \in \stackrel{o}{W^{2 m}}(\Omega)
$$

and

$$
v \in \stackrel{o}{W} 2 m(\Omega)
$$

(e) The essential spectrum $\sigma_{\text {ess }}\left(T_{\text {Har }}\right)=\{0\}$ and so is non-empty.

Remark 2.2. In general, we cannot exclude the possibility that the eigenvalue sequence $\left(\lambda_{n}: n \in \mathbb{N}\right)$ accumulates at both $+\infty$ and $-\infty$. If however $T_{0}$ is a positive operator, i.e. if $\left\langle T_{0} v, v\right\rangle>0$ for all $v \in \stackrel{\circ}{W}^{2 m}(\Omega)$ with $v \neq 0$, then the eigenvalues of the problem (2.1) and (2.2) are all positive and $\lim _{n \rightarrow \infty} \lambda_{n}=\infty$.

We break down the proof of Theorem 2.1] into several lemmata.

Lemma 2.3. Let $T_{\text {Dir }}$ denote the Dirichlet operator defined in (1.23). Then there exist positive numbers $c_{1}, c_{2}$ such that (recall that $\|\cdot\|_{2 m}$ denotes the norm in the Hilbert space $\left.W^{2 m}(\Omega)\right)$

$$
c_{1}\|u\|_{2 m} \leq\left\|T_{D i r} u\right\| \leq c_{2}\|u\|_{2 m} \text { for all } u \in D\left(T_{D i r}\right) .
$$

Proof. The existence of the number $c_{2}$ follows from the fact that $T_{D i r} u:=A(\cdot, D) u$ as in (1.23), and that $T_{D i r}$ is generated by a differential expression $A(\cdot, D)$ of order $2 m$ with bounded coefficients in the compact set $\bar{\Omega}$.

Due to our assumption $(c)$ of Condition 1.1 and as a consequence of the fact that the map in (1.5) is a Fredholm operator of index 0, the mapping $T_{D i r}: D\left(T_{D i r}\right) \rightarrow$ $L_{2}(\Omega)$ is one-to-one and onto; see (1.7). Further, since $D\left(T_{D i r}\right)$, endowed with the inner-product $\langle\cdot, \cdot\rangle_{2 m}$, is a Hilbert space, the inverse mapping theorem ensures the boundedness of $T_{\text {Dir }}^{-1}: L_{2}(\Omega) \rightarrow W^{2 m}(\Omega)$, and hence the existence of the number $c_{1}$.

Since $T_{0}=\left.T_{D i r}\right|_{W^{2 m}(\Omega)}$, Lemma 2.3 shows, in particular, that

$$
(v, \widetilde{v}):=\left\langle T_{0} v, T_{0} \widetilde{v}\right\rangle \text { for all } v, \widetilde{v} \in \stackrel{\circ}{W}^{2 m}(\Omega)
$$

defines an inner product $(\cdot, \cdot)$ on $\stackrel{\mathrm{o}}{W}^{2 m}(\Omega)$, where the corresponding norm $|v|:=$ $\sqrt{(v, v)}$ is equivalent to the norm $\|\cdot\|_{2 m}$.

Lemma 2.4. The eigenvalue problem (2.1) and (2.2) has $a(\cdot, \cdot)$-orthonormal and complete sequence of eigenfunctions $\left(v_{n} \in \stackrel{\circ}{W^{2 m}}(\Omega): n \in \mathbb{N}\right)$. The corresponding eigenvalues $\left(\lambda_{n}: n \in \mathbb{N}\right)$ are real and non-zero, and have finite multiplicity; also $\lim _{n \rightarrow \infty}\left|\lambda_{n}\right|=\infty$.

Proof. For each fixed $g \in L_{2}(\Omega)$, the linear functional $F_{g}: \stackrel{\circ}{W^{2 m}}(\Omega) \rightarrow \mathbb{C}$, defined by $F_{g}[\varphi]:=\left\langle T_{0} \varphi, g\right\rangle$, is bounded since

$$
\left|F_{g}[\varphi]\right| \leq\left\|T_{0} \varphi\right\|\|g\|=|\varphi|\|g\| \text { for all } \varphi \in \stackrel{\circ}{W^{2 m}}(\Omega) .
$$


The Riesz representation lemma for bounded linear functionals in Hilbert space then provides a unique element $v=: K_{0} g \in \stackrel{\mathrm{o}}{W}^{2 m}(\Omega)$, for all $g \in L_{2}(\Omega)$, such that

$$
(\varphi, v)=F_{g}[\varphi] \text { for all } \varphi \in \stackrel{\circ}{W}{ }^{2 m}(\Omega) .
$$

This defines a linear operator $K_{0}: L_{2}(\Omega) \rightarrow \stackrel{\circ}{W}{ }^{2 m}(\Omega)$ satisfying

$$
\left(\varphi, K_{0} g\right)=\left\langle T_{0} \varphi, g\right\rangle \text { for all } g \in L_{2}(\Omega) \text { and } \varphi \in \stackrel{\circ}{W^{2 m}}(\Omega) \text {. }
$$

Also $K_{0}$ is bounded since, for each $g \in L_{2}(\Omega)$, the choice $\varphi:=K_{0} g$ in (2.5) gives

$$
\left|K_{0} g\right|^{2}=\left\langle T_{0}\left(K_{0} g\right), g\right\rangle \leq\left\|T_{0}\left(K_{0} g\right)\right\|\|g\|=\left|K_{0} g\right|\|g\|,
$$

whence $\left|K_{0} g\right| \leq\|g\|$.

Furthermore, $\left.K_{0}\right|_{W^{2 m}(\Omega)}$ is $(\cdot, \cdot)$-symmetric, because (2.5) and the $\langle\cdot, \cdot\rangle$-symmetry of $T_{0}$ yield, for all $g, h \in \stackrel{\circ}{W^{2 m}}(\Omega)$,

$$
\left(h, K_{0} g\right)=\left\langle T_{0} h, g\right\rangle=\left\langle h, T_{0} g\right\rangle=\overline{\left\langle T_{0} g, h\right\rangle}=\overline{\left(g, K_{0} h\right)}=\left(K_{0} h, g\right) .
$$

Finally, the embedding $E: \stackrel{\circ}{W^{2 m}}(\Omega) \hookrightarrow L_{2}(\Omega)$ is compact due to the SobolevKondrachev-Rellich embedding theorem. Altogether, we obtain

$$
K:=K_{0} \circ E: \stackrel{\circ}{W^{2 m}}(\Omega) \rightarrow \stackrel{\circ}{\rightarrow}{ }^{2 m}(\Omega)
$$

is compact and $(\cdot, \cdot)$-symmetric.

The spectral theorem for compact symmetric operators in Hilbert space therefore yields a $(\cdot, \cdot)$-orthonormal and complete sequence $\left(v_{n}: n \in \mathbb{N}\right)$ of eigenfunctions of $K$, corresponding to a sequence of real eigenvalues $\left(\mu_{n}: n \in \mathbb{N}\right)$, each of finite multiplicity, and further $\lim _{n \rightarrow \infty} \mu_{n}=0$.

Since $K v_{n}=\mu_{n} v_{n}$ with $v_{n} \in \stackrel{\circ}{W}^{2 m}(\Omega)$, the results (2.4) and (2.5) imply, for all $n \in \mathbb{N}$ and $\varphi \in \stackrel{\circ}{W}^{2 m}(\Omega)$,

$$
\mu_{n}\left\langle T_{0} v_{n}, T_{0} \varphi\right\rangle=\mu_{n}\left(v_{n}, \varphi\right)=\left(K v_{n}, \varphi\right)=\left\langle v_{n}, T_{0} \varphi\right\rangle .
$$

This implies $\mu_{n} \neq 0$ for all $n \in \mathbb{N}$, since otherwise (2.6) would give (see Theorem [1.6 above)

$$
v_{n} \in\left[T_{0}\left(\stackrel{\circ}{W^{2 m}}(\Omega)\right)\right]^{\perp}=\stackrel{A}{L}(\Omega)
$$

whence $v_{n}=0$ since also $v_{n} \in \stackrel{\circ}{W}^{2 m}(\Omega)$. This result contradicts the fact that the eigenfunction $v_{n} \neq 0$.

If now we define $\lambda_{n}:=\mu_{n}^{-1}$ for all $n \in \mathbb{N}$, then the proof of Lemma 2.4 is complete.

Lemma 2.5. With $\left(\lambda_{n}: n \in \mathbb{N}\right)$ and $\left(v_{n}: n \in \mathbb{N}\right)$ denoting the sequences of eigenvalues and eigenfunctions, respectively, provided by Lemma 2.4 define the sequence

$$
u_{n}:=T_{0} v_{n} \text { for all } n \in \mathbb{N} \text {. }
$$

\section{Then}

(a) $u_{n} \in D\left(T_{\text {Har }}\right)=\stackrel{o}{W^{2 m}}(\Omega)+\stackrel{A}{L_{2}}(\Omega)$ and $T_{\text {Har }} u_{n}=\lambda_{n} u_{n}$ for all $n \in \mathbb{N}$. 
(b) The sequence $\left(u_{n}: n \in \mathbb{N}\right)$ is a $\langle\cdot, \cdot\rangle$-orthonormal and complete system in the Hilbert space

$$
T_{0}\left(\stackrel{o}{W^{2 m}}(\Omega)\right) \subset L_{2}(\Omega) .
$$

(c) For each $n \in \mathbb{N}$ the finite multiplicity of $\lambda_{n}$, as an eigenvalue of the boundary value problem (2.1) and (2.2), is equal to the multiplicity of $\lambda_{n}$ as an eigenvalue of the operator $T_{H a r}$.

Proof. We give the proofs for the items $(a),(b)$ and $(c)$ as follows:

(a) For $n \in \mathbb{N}$, the formulation (2.1) and (2.2) shows that

$$
\left\langle T_{0} v_{n}-\lambda_{n} v_{n}, T_{0} \varphi\right\rangle=0 \text { for all } \varphi \in \stackrel{\circ}{W}^{2 m}(\Omega),
$$

whence, from Theorem 1.6

$$
T_{0} v_{n}-\lambda_{n} v_{n} \in\left[T_{0}(\stackrel{\circ}{W} 2 m(\Omega))\right]^{\perp}=\stackrel{A}{L_{2}}(\Omega) .
$$

Therefore

$$
u_{n}=T_{0} v_{n}=\lambda_{n} v_{n}+\left(T_{0} v_{n}-\lambda_{n} v_{n}\right) \in \stackrel{\circ}{W}{ }^{2 m}(\Omega)+\stackrel{A}{L}_{2}(\Omega)=D\left(T_{\text {Har }}\right) .
$$

So $T_{0} v_{n}-\lambda_{n} v_{n} \in \stackrel{A}{L_{2}}(\Omega)$ and thus $T_{\text {Har }}\left(T_{0} v_{n}-\lambda_{n} v_{n}\right)=0$ implies $T_{\text {Har }} u_{n}=\lambda_{n} u_{n}$ by (2.7), since $T_{H a r} v_{n}=T_{0} v_{n}=u_{n}$.

(b) For all $k, n \in \mathbb{N}$,

$$
\left\langle u_{n}, u_{k}\right\rangle=\left\langle T_{0} v_{n}, T_{0} v_{k}\right\rangle=\left(v_{n}, v_{k}\right)=\delta_{n, k} .
$$

Moreover, $u_{n}=T_{0} v_{n} \in T_{0}\left(\stackrel{\circ}{W^{2 m}}(\Omega)\right)$. Then to show the asserted completeness property, let $f=T_{0} g \in T_{0}\left(\stackrel{\circ}{W^{2 m}}(\Omega)\right)$ satisfy $\left\langle f, u_{n}\right\rangle=0$ for all $n \in \mathbb{N}$, which gives

$$
0=\left\langle f, u_{n}\right\rangle=\left\langle T_{0} g, T_{0} v_{n}\right\rangle=\left(g, v_{n}\right) \text { for all } n \in \mathbb{N},
$$

whence the $(\cdot, \cdot)$-completeness of the sequence $\left(v_{n}: n \in \mathbb{N}\right)$ implies $g=0$ and thus, $f=0$.

(c) Let $\lambda=\lambda_{n_{1}}=\cdots=\lambda_{n_{k}} \neq 0$ be an eigenvalue of (2.1) and (2.2) with multiplicity $k \in \mathbb{N}$. Since $T_{0}$ is one-to-one, (2.7) shows that $u_{n_{1}}, \ldots, u_{n_{k}}$ are linearly independent eigenfunctions of $T_{\text {Har }}$ corresponding to the eigenvalue $\lambda$. Thus the multiplicity of $\lambda$ as an eigenvalue of $T_{H a r}$ is at least $k$.

It remains to show that for each $u \in D\left(T_{\text {Har }}\right)$ satisfying $T_{H a r} u=\lambda u$ we have $u \in \operatorname{span}\left\{u_{n_{1}}, \ldots, u_{n_{k}}\right\}$. Indeed, by the definition of $D\left(T_{\text {Har }}\right)$, such an element $u$ can be represented as $u=v+g$ with $v \in \stackrel{\circ}{W}{ }^{2 m}(\Omega)$ and $g \in \stackrel{A}{L_{2}}(\Omega)$. Thus, $u=\lambda^{-1} T_{\text {Har }} u=\lambda^{-1} T_{0} v \in T_{0}\left(\stackrel{\circ}{W^{2 m}}(\Omega)\right)$, whence part $(b)$ above yields

$$
u=\sum_{t=1}^{\infty}\left\langle u, u_{t}\right\rangle u_{t} .
$$

Since $\left\langle u, u_{t}\right\rangle=0$ for all $t$ different to every $n_{1}, n_{2}, \ldots, n_{k}$, this result implies that $u \in \operatorname{span}\left\{u_{n_{1}}, \ldots, u_{n_{k}}\right\}$.

We can now complete the proof of Theorem[2.1. 
Proof. First recall that $T_{0}\left(\stackrel{\circ}{W}{ }^{2 m}(\Omega)\right)$ is a closed subspace of $L_{2}(\Omega)$, and then, using Theorem 1.6 above,

$$
L_{2}(\Omega)=T_{0}(\stackrel{\circ}{W}=\stackrel{\perp}{2 m}(\Omega)) \stackrel{A}{\oplus} L_{2}(\Omega) .
$$

By Theorem 1.6, (1.39) and the separability of ${ }^{A} L_{2}(\Omega) \subset L_{2}(\Omega)$ we can choose a $\langle\cdot, \cdot\rangle$-orthonormal and complete system $\left(\widetilde{u}_{n}: n \in \mathbb{N}\right)$ in $\stackrel{A}{L}(\Omega)$.

In fact, since $\operatorname{Ker}\left(T_{\text {Har }}\right)=\stackrel{A}{L_{2}}(\Omega)$ (see (1.28) above) $\widetilde{u}_{n}$ is an eigenfunction for the eigenvalue 0 of $T_{\text {Har }}$, for each $n \in \mathbb{N}$. Thus 0 is an eigenvalue of countable multiplicity and so the essential spectrum satisfies $\sigma_{e s s}\left(T_{\text {Har }}\right) \neq \emptyset$. Since all the other eigenvalues have finite multiplicity it follows that $\sigma_{\text {ess }}\left(T_{\text {Har }}\right)=\{0\}$, as asserted in conclusion $(e)$.

From (2.8) and (b) of Lemma 2.5 and the above remark, we conclude that the (interlacing) union of the systems $\left(u_{n}: n \in \mathbb{N}\right)$ and $\left(\widetilde{u}_{n}: n \in \mathbb{N}\right)$ forms a $\langle\cdot, \cdot \cdot\rangle$ orthonormal and complete system in the whole of $L_{2}(\Omega)$.

Using the Lemmata 2.4] and 2.5, we find that all the required conclusions $(a)$ to (e) have now been demonstrated, so that the proof of Theorem[2.1 is now complete.

\section{A STRONG FORMUlation OF THE PROBLEM}

In this short section, we give further consideration to the auxiliary eigenvalue problem (2.1) and (2.2) which plays an important role in determining the spectrum of the operator $T_{\text {Har }}$. In particular we prove the equivalence of this problem to the $4 m$-th order boundary value problem given by the differential equation

$$
T_{H a r}\left(T_{0} v\right)=\lambda T_{0} v
$$

with generalized boundary conditions

$$
v \in D\left(T_{H a r} T_{0}\right) .
$$

Lemma 3.1. The eigenvalue problems (2.1) and (2.2), and (3.1) and (3.2) have the same eigenpairs.

Proof. Let $(v, \lambda)$ denote an eigenpair of (3.1) and (3.2). Since $T_{H a r} \subset T_{1}=T_{0}^{*}$ the boundary condition (3.2) yields $v \in D\left(T_{0}\right)=\stackrel{\circ}{W}{ }^{2 m}(\Omega), T_{0} v \in D\left(T_{0}^{*}\right)$, whence (3.1) gives

$$
\left\langle T_{0}^{*} T_{0} v, \varphi\right\rangle=\lambda\left\langle T_{0} v, \varphi\right\rangle \text { for all } \varphi \in \stackrel{\circ}{W^{2 m}}(\Omega) .
$$

Exploiting the properties of the adjoint operator on the left-hand side, and the symmetry of $T_{0}$ on the right-hand side, we deduce that $(v, \lambda)$ is an eigenpair of the problem (2.1) and (2.2).

Conversely, let $(v, \lambda)$ be an eigenpair of the problem (2.1) and (2.2). Then

$$
\left\langle T_{0} v-\lambda v, T_{0} \varphi\right\rangle=0 \text { for all } \varphi \in \stackrel{\circ}{W}^{2 m}(\Omega),
$$

whence $T_{0} v-\lambda v \in\left[T_{0}\left(\stackrel{\circ}{W^{2 m}}(\Omega)\right)\right]^{\perp}=\stackrel{A}{L_{2}}(\Omega)$ from Theorem 1.6 above. 
Consequently,

$$
T_{0} v=\lambda v+\left(T_{0} v-\lambda v\right) \in \stackrel{\circ}{W^{2 m}}(\Omega)+\stackrel{A}{L}(\Omega)=D\left(T_{H a r}\right)
$$

implying that $v \in D\left(T_{H a r} T_{0}\right)$ and, by symmetry of $T_{\text {Har }}$,

$$
\left\langle T_{0} v, T_{0} \varphi\right\rangle=\left\langle T_{0} v, T_{H a r} \varphi\right\rangle=\left\langle T_{H a r} T_{0} v, \varphi\right\rangle \text { for all } \varphi \in \stackrel{\circ}{W^{2 m}}(\Omega) .
$$

Therefore, we obtain from (2.1) and (2.2), using the symmetry of $T_{0}$ on the righthand side of 2.1, that

$$
\left\langle T_{H a r} T_{0} v-\lambda T_{0} v, \varphi\right\rangle=0 \text { for all } \varphi \in \stackrel{\circ}{W^{2 m}}(\Omega),
$$

whence the density of $\stackrel{\circ}{W}^{2 m}(\Omega)$ in $L_{2}(\Omega)$ provides the assertion.

Remark 3.2. A "natural" Hilbert space for studying the eigenvalue problem (3.1) and (3.2) is $\stackrel{\circ}{W}^{2 m}(\Omega)$ endowed with the inner product $(\cdot, \cdot)$ from (2.4), because that inner product is generated by the right-hand side of (3.1). In this setting problem (3.1) and (3.2) has an orthonormal and complete system of eigenfunctions, as readily follows from Lemmata 2.4 and 3.1. Thus, Lemma 3.1 gives the full spectral equivalence of the boundary value problems (2.1) and (2.2), and (3.1) and (3.2).

Remark 3.3. Since $T_{H a r} \subset T_{1}=T_{0}^{*}$, the eigenvalue problem

$$
T_{1} T_{0} v=\lambda T_{0} v
$$

with generalized boundary conditions

$$
v \in D\left(T_{1} T_{0}\right)
$$

is also equivalent to (2.1) and (2.2), and to (3.1) and (3.2).

Furthermore, another phraseology for the eigenvalue problem (2.1) and (2.2) is indicated by

$$
T_{0} v-\lambda v \in\left[T_{0}\left(\stackrel{\circ}{W^{2 m}}(\Omega)\right)\right]^{\perp}=\stackrel{A}{L_{2}}(\Omega), \text { with } v \in \stackrel{\circ}{W}{ }^{2 m}(\Omega)
$$

or equally well

$$
T_{0} v=\lambda v\left(\bmod \stackrel{A}{L}{ }_{2}(\Omega)\right) \text {, with } v \in \stackrel{\circ}{W^{2 m}}(\Omega) .
$$

Example 3.4. Let $m=1$ and $A(\cdot, D)=-\Delta$, where $\Delta$ is the Laplacian differential expression in $\mathbb{E}^{2}$. Then, the boundary value problems (2.1) and (2.2), and (3.1) and (3.2) are the weak and strong formulation, respectively, of the well-known buckling plate problem [14, Chapter 9, Sections 62 to 65]

$$
\langle\Delta v, \Delta \varphi\rangle=\lambda\langle v,-\Delta \varphi\rangle \text { for all } \varphi \in \stackrel{\circ}{W^{2 m}}(\Omega)
$$

with

$$
v \in \stackrel{\circ}{W}^{2 m}(\Omega)
$$

Remark 3.5. In general there appears to be no connection of $T_{\text {Har }}$ with the Krein extension (see [2, Example 5.3]) or with the Friedrichs extension of $T_{0}$, since both of these extensions maintain the lower bound of $T_{0}$. However, for example in the case when $A(\cdot, D)=-\Delta, T_{0}$ has a positive lower bound whilst $T_{H a r}$ has zero as an eigenvalue. 


\section{Boundary Behaviour in $D\left(T_{\text {Har }}\right)$}

In accordance with the introductory Section 1 above, we now consider the classical Laplacian differential expression

$$
\Delta:=\sum_{j=1}^{r} D_{j}^{2} \text { with } D_{j}=\frac{\partial}{\partial x_{j}}
$$

in a bounded region $\Omega$, with smooth boundary $\partial \Omega$, in the real Euclidean space $\mathbb{E}^{r}$ $(r \geq 2)$, as described in terms of the real cartesian coordinates $\mathbf{x}=\left(x_{1}, x_{2}, \ldots, x_{r}\right)$. We further consider the corresponding linear operator, with the conventional negative sign,

$$
A: f \rightarrow-\Delta f
$$

on its classical domain

$$
D(A):=C_{0}^{\infty}(\Omega),
$$

which is interpreted as a dense linear manifold of the complex Hilbert space $L_{2}(\Omega)$.

Then the operator $A$ on $D(A)$, as defined in (4.2) and (4.3), satisfies the general Condition 1.1 of Section 1, and $A$ has infinitely many self-adjoint extensions (see [8, Theorem 2.1]), including the classical Dirichlet and Neumann extensions, and also unusual extensions such as the Harmonic operator

$$
T_{H a r} f:=-\Delta f
$$

for

$$
f \in D\left(T_{\text {Har }}\right)=\stackrel{\circ}{W}{ }^{2}(\Omega) \dot{+} \stackrel{\Delta}{L}_{2}(\Omega)
$$

as in (1.29) to (1.36) of Section 1. As remarked earlier the domain $D\left(T_{\text {Har }}\right)$ is a linear submanifold of $L_{2}(\Omega)$, constituted as the direct sum of the two submanifolds

$$
\stackrel{\circ}{W}^{2}(\Omega)=\left\{f \in W^{2}(\Omega):\left.f\right|_{\partial \Omega}=0,\left.\frac{\partial f}{\partial \mathbf{n}}\right|_{\partial \Omega}=0\right\}
$$

and

$$
\stackrel{\Delta}{L}_{2}(\Omega)=\left\{f \in L_{2}(\Omega): f \in C^{\infty}(\Omega) \text { and } \Delta f=0 \text { in } \Omega\right\} .
$$

We omit any further discussion of $\stackrel{\circ}{W^{2}}(\Omega)$, which is defined within $W^{2}(\Omega)$ by the familiar boundary conditions

$$
\left.f\right|_{\partial \Omega}=0,\left.\frac{\partial f}{\partial \mathbf{n}}\right|_{\partial \Omega}=0
$$

and henceforth concentrate on the relatively unfamiliar manifold $\stackrel{\Delta}{L}_{2}(\Omega)$, the set of all harmonic functions in $L_{2}(\Omega)$. However, it is known (see Remark 1.5 above) that $\stackrel{\Delta}{L}_{2}(\Omega)$ is a closed Hilbert subspace of $L_{2}(\Omega)$. We present several interesting examples of functions in $\stackrel{\Delta}{L}_{2}(\Omega)$, under the simplest kinds of geometric conditionssay $\Omega$ is an open disk in $\mathbb{E}^{2}$ or an open ball in $\mathbb{E}^{3}$-in order to demonstrate the unavoidable obstacles to specifying or characterizing the functions of $\stackrel{\Delta}{L}_{2}(\Omega)$ through their boundary behavior. In particular, we offer examples of functions in $\stackrel{\Delta}{L}_{2}(\Omega)$ 
which do not belong to $W^{2}(\Omega)$, and which have highly singular and pathological boundary values-in fact, no describable boundary values in any conventional sense.

Accordingly, we first fix the region $\Omega$ as the open unit disk centered at the origin in the complex plane $\mathbb{C}$ (topologically $\mathbb{E}^{2}$ ),

$$
\Omega=\{z \in \mathbb{C}:|z|<1\},
$$

where the complex number

$$
z=x+i y \text { or } z=r \exp (i \theta),
$$

in terms of the real cartesian coordinates $(x, y)$ or the polar coordinates $(r, \theta)$, as usual.

Consider the complex linear space $\mathcal{H}(\Omega)$ of all holomorphic functions in the open disk $\Omega$, that is,

$$
\mathcal{H}(\Omega):=\{F: \Omega \rightarrow \mathbb{C}: F \text { is holomorphic in } \Omega\} .
$$

Hence each function $F \in \mathcal{H}(\Omega)$ has an absolutely convergent power series, with complex coefficients $\left\{a_{n}: n \in \mathbb{N}_{0}\right\}$,

$$
F(z)=\sum_{n=0}^{\infty} a_{n} z^{n} \text { for }|z|<1,
$$

or equally well

$$
F(r \exp (i \theta))=\sum_{n=0}^{\infty} a_{n} r^{n} \exp (i n \theta) \text { for } 0 \leq r<1 \text { and } 0 \leq \theta<2 \pi .
$$

Since we may write $F(z)=u(x, y)+i v(x, y)$ for real-valued harmonic functions $u, v \in C^{\infty}(\Omega)$, we observe that

$$
F \in C^{\infty}(\Omega) \text { and } \Delta F=0 \text { in } \Omega .
$$

Thus we are led to seek holomorphic functions $F \in \mathcal{H}(\Omega)$ that also belong to the Hilbert space $L_{2}(\Omega)$, that is,

$$
\iint_{\Omega}|F(z)|^{2} d x d y=\iint_{\Omega}\left(u(x, y)^{2}+v(x, y)^{2}\right) d x d y<\infty,
$$

so $u, v \in L_{2}(\Omega)$.

Definition 4.1. The Bergman Hilbert space $B_{2}(\Omega)$ (see [5]) is defined by

$$
B_{2}(\Omega):=\mathcal{H}(\Omega) \cap L_{2}(\Omega),
$$

which is known to be a Hilbert subspace of $L_{2}(\Omega)$; see [16]. Hence $B_{2}(\Omega)$ (and the complex conjugate space $\left.\overline{B_{2}(\Omega)}\right)$ are closed subspaces of $L_{2}(\Omega)$, which is itself a Hilbert subspace of $L_{2}(\Omega)$. As such, using harmonic conjugates, we can demonstrate that

$$
\stackrel{\Delta}{L}_{2}(\Omega)=\operatorname{span}\left\{B_{2}(\Omega), \overline{B_{2}(\Omega)}\right\} .
$$

Let $F(z)=\sum_{n=0}^{\infty} a_{n} z^{n} \in \mathcal{H}(\Omega)$. Then for each positive radius $R<1$,

$$
\int_{0}^{R} \int_{0}^{2 \pi}|F(z)|^{2} r d r d \theta=\int_{0}^{R} 2 \pi \sum_{n=0}^{\infty}\left|a_{n}\right|^{2} r^{2 n+1} d r=\pi \sum_{n=0}^{\infty} \frac{\left|a_{n}\right|^{2}}{n+1} R^{2 n+2}
$$


Accordingly, for $F \in \mathcal{H}(\Omega)$ we obtain the convenient test for $F \in B_{2}(\Omega)$ :

$$
F \in B_{2}(\Omega) \text { if and only if } \sum_{n=0}^{\infty} \frac{\left|a_{n}\right|^{2}}{n+1}<\infty .
$$

The next example demonstrates that the boundary behaviour, with respect to radial limits, of functions $F \in B_{2}(\Omega)$ can be notoriously difficult and unpredictable.

Example 4.2. Define the holomorphic function $F \in \mathcal{H}(\Omega)$ by

$$
F(z):=\sum_{n=0}^{\infty} z^{2^{n}}=z+z^{2}+z^{4}+z^{8}+\cdots
$$

which converges absolutely for each $|z|<1$ and

$$
\sum_{k=0}^{\infty} \frac{\left|a_{k}\right|^{2}}{k+1}=\sum_{n=0}^{\infty} \frac{1}{2^{n}+1}<\infty,
$$

and so $F \in B_{2}(\Omega) \subset \stackrel{\Delta}{L}_{2}(\Omega)$, as desired.

Clearly the series (4.19) converges at no point on the boundary circle $\partial \Omega$, so we try to establish boundary values for $F$ on $\partial \Omega$ by means of radial limits for each ray, fixed by $\theta \in[0,2 \pi)$, as in

$$
\lim _{r \succ 1} F(r \exp (i \theta))=\lim _{r \nearrow^{1}} \sum_{n=0}^{\infty} \exp \left(i 2^{n} \theta\right) r^{2^{n}} .
$$

First let $\theta=p q^{-1} \pi$, for positive integers $p<q$, and take $q=2^{t}$ for some $t \in \mathbb{N}$. Then $\exp \left(i 2^{n} \theta\right)=\exp \left(i 2^{n-t} p \pi\right)=1$, for all $n>t$. Hence for each $\theta$ of a countable but dense set of rays $\Theta \subset[0,2 \pi)$,

$$
\lim _{r \nearrow 1}|F(r \exp (i \theta))|=+\infty .
$$

In greater detail we compute, for each $N \in \mathbb{N}$ with $N>t+1$,

$$
|F(r \exp (i \theta))| \geq \sum_{n=t+1}^{N} r^{2^{n}}-\sum_{n=0}^{t} 1
$$

and thus

$$
|F(r \exp (i \theta))| \geq \sum_{n=t+1}^{\infty} r^{2^{n}}-(t+1) .
$$

Since $\sum_{n=t+1}^{\infty} r^{2^{n}}$ tends to $+\infty$ as $r \nearrow 1$, we conclude that

$$
\lim _{r \nearrow 1}|F(r \exp (i \theta))|=+\infty \text { for all } \theta \in \Theta .
$$

For rays with $\theta \notin \Theta$ we can apply the famous Tauberian theorems of Hardy and Littlewood; see [3] and [11, Theorems 90 and 114]. Namely, if there exists $\theta \in[0,2 \pi)$ for which

$$
\lim _{r \nearrow 1} F(r \exp (i \theta))=s,
$$

where $s \in \mathbb{C}$, then, with convergence in $\mathbb{C}$,

$$
\sum_{n=0}^{\infty} \exp \left(i 2^{n} \theta\right)=s
$$


However, 4.23) is impossible since $\left|\exp \left(i 2^{n} \theta\right)\right|=1$ for all $n \in \mathbb{N}$. Hence for each $\theta \in[0,2 \pi)$ the finite radial limit of (4.22) cannot exist; therefore there is no finite limit (as $r / 1$ ) in (4.21) defining a finite value for $F$ on $\partial \Omega$.

Therefore we conclude, for this example, that $F$ defined in (4.19) cannot be assigned radial values $f(\theta)$, say, such that $f \in L_{1}(\partial \Omega)$.

A personal communication from J.M. Anderson [4] remarks (see [11, Theorem 116]) that for the example

$$
G(z)=\sum_{n=0}^{\infty} n z^{2^{n}}
$$

which belongs to $\stackrel{\Delta}{L}_{2}(\Omega)$ as before,

$$
\limsup _{r \nearrow 1}|G(r \exp (i \theta))|=+\infty
$$

for every $\theta \in[0,2 \pi)$. Remark 4.4 also applies here.

Remark 4.3. As an interesting consequence of these arguments, it follows that $F$ of (4.19) does not belong to any Hardy space $H^{p}$, for any real $p \geq 1$; see [12] and [17. Thus there is no possibility of defining a boundary function $f \in L_{1}[0,2 \pi)$ as the $L_{1}$-limit as $r \nearrow 1$ of the functions $f_{r}:[0,2 \pi) \rightarrow \mathbb{C}$, where $f_{r}(\theta):=F(r \exp (i \theta))$ for $\theta \in[0,2 \pi)$ (and all these functions are defined on $[0,2 \pi)$, noting that this domain can be identified with the boundary circle $\partial \Omega$ ).

Remark 4.4. For $F \in L_{2}(\Omega)$, as defined in (4.19) of Example 4.2 we note that, for $z \in \Omega$,

$$
F^{\prime}(z)=1+2 z+4 z^{3}+8 z^{7}+\cdots
$$

and that the test indicated in (4.18) shows that the derivative $F^{\prime} \notin L_{2}(\Omega)$. We conclude that at least one of the first-order cartesian partial derivatives of real and imaginary parts of $F$ is not in $L_{2}(\Omega)$; hence $F \notin W^{1}(\Omega)$. Therefore $\stackrel{\Delta}{L}_{2}(\Omega)$ is not contained in the Sobolev space $W^{1}(\Omega)$, and furthermore the trace theory does not apply to $F$.

We next turn to the case where $\Omega$ is the unit open ball centered at the origin in $\mathbb{E}^{3}$. Thus

$$
\Omega=\left\{\mathbf{x}=(x, y, z): 0 \leq x^{2}+y^{2}+z^{2}<1\right\},
$$

or in terms of polar coordinates

$$
\Omega=\{(r, \theta, \varphi): r \in[0,1), \theta \in[-\pi / 2, \pi / 2], \varphi \in[0,2 \pi]\}
$$

where, as usual,

$$
x=r \cos (\theta) \cos (\varphi), y=r \cos (\theta) \sin (\varphi), z=r \sin (\theta) .
$$

Example 4.5. We examine the real function

$$
F: \Omega \rightarrow \mathbb{R} \text { given by } F(x, y, z)=\left[(x+1)^{2}+y^{2}+z^{2}\right]^{-1 / 2},
$$

which is the Newtonian potential for a pole $P_{0}$ at $x=-1, y=z=0$, or

$$
P_{0}=(-1,0,0) \text {. }
$$

For each point $P=(x, y, z) \in \Omega$, we also write

$$
F(P)=\left|P-P_{0}\right|^{-1} \text {. }
$$


Here $\left|P-P_{0}\right|$ is the euclidean distance between $P=(x, y, z) \in \Omega$ and $P_{0} \in \partial \Omega$.

Certainly $F \in C^{\infty}(\Omega)$ and $\Delta F \equiv 0$ in $\Omega$. By straightforward calculation, using polar coordinates centered at $P_{0}$, it can be verified that $F \in L_{2}(\Omega)$ (although $\left.F \notin W^{1}(\Omega)\right)$; hence $F \in \stackrel{\Delta}{L}_{2}(\Omega)$ as required.

In order to compute the boundary function $f=\left.F\right|_{\partial \Omega}$, we can either use radial limits as $r \nearrow 1$, or else note that $F$ has a continuous extension to $\bar{\Omega} \backslash P_{0}$ which we call $f$. Again direct calculations verify that $f \in L_{1}(\partial \Omega)$, yet $f \notin L_{2}(\partial \Omega)$.

As a final comment on $F=\left|P-P_{0}\right|^{-1}$ as in (4.30), we can define (in terms of the usual polar coordinates (4.27)

$$
f_{r}(\theta, \varphi):=F(r, \theta, \varphi) \text { for all } r \in[0,1),
$$

and interpret $f_{r}$ as a real function on the sphere $S^{2}$. In this sense we can further verify that

$$
\lim _{r \nearrow 1} f_{r}=f
$$

with convergence in $L_{1}(\partial \Omega)$, in fact uniform convergence on the exterior of each fixed neighborhood of $P_{0} \in \partial \Omega$.

Example 4.6. As in the prior Example 4.5 consider the unit open ball $\Omega \subset \mathbb{E}^{3}$ with the boundary sphere $S^{2}=\partial \Omega$. We shall utilize the function $F$ of (4.30) to define a function $F^{*} \in \stackrel{\Delta}{L}_{2}(\Omega)$, which has a dense set of singularities on the boundary sphere $S^{2}=\partial \Omega$.

To define $F^{*} \in \stackrel{\Delta}{L}_{2}(\Omega)$ choose a countable set of (distinct) points, dense in the boundary space $S^{2}=\partial \Omega$, say

$$
P_{k} \in \partial \Omega \text { for } k \in \mathbb{N}_{0}=\{0,1,2,3, \ldots\}
$$

and fix $P_{0}=(-1,0,0)$ as in (4.29) of Example 4.5. For each $k \in \mathbb{N}_{0}$ define the corresponding harmonic function

$$
F(P, k):=\left|P-P_{k}\right|^{-1} \text { for all } P \in \Omega .
$$

Then $F(P, 0)=F(P)$ as in Example 4.5 above, and we sometimes denote $F(\cdot, k)$ by $F(P, k)$ or $F(x, y, z, k)$ (or even $F(r, \theta, \varphi, k)$, as is convenient).

Lemma 4.7. For each $F(\cdot, k)$, with $k \in \mathbb{N}_{0}$,

$$
\|F(\cdot, k)\|_{L_{2}(\Omega)}=\|F(\cdot, 0)\|_{L_{2}(\Omega)} .
$$

Further, for each compact ball $\overline{B_{R}}(0)$ of radius $R \in(0,1)$ about the origin,

$$
\sup \left\{|F(P, k)|: P \in \overline{B_{R}}(0)\right\}=\sup \left\{|F(P, 0)|: P \in \overline{B_{R}}(0)\right\} .
$$

Proof. For each $k \in \mathbb{N}_{0}$ there exists a rigid rotation of $\bar{\Omega}$ which carries $P_{k}$ to $P_{0}$, and hence $F(\cdot, k)$ into $F(\cdot, 0)$. Since the norm for $L_{2}(\Omega)$ and the uniform norm on $\overline{B_{R}}(0)$ are preserved under such rigid rotations, the required equalities in the lemma now follow.

Definition 4.8. Define the real positive function $F^{*}$ in $\Omega$ by

$$
F^{*}(P):=\sum_{k=0}^{\infty} \frac{1}{k !} F(P, k) \text { for all } P \in \Omega .
$$


Theorem 4.9.

$$
F^{*} \in \stackrel{\Delta}{L}_{2}(\Omega)
$$

Proof. By (4.36) we observe that, for $P \in \overline{B_{R}}(0)$,

$$
\left|\sum_{k=m}^{n} \frac{1}{k !} F(P, k)\right| \leq \sup \left\{|F(P, 0)|: P \in \overline{B_{R}}(0)\right\} \sum_{k=m}^{n} \frac{1}{k !} \text { for all } m<n \text { in } \mathbb{N}_{0} .
$$

Hence the partial sums of the infinite series (4.37) form a Cauchy sequence in the complete metric space $C\left(\overline{B_{R}}(0)\right)$, and hence the series converges uniformly on $\overline{B_{R}}(0)$. Therefore $F^{*}$ in (4.37) is well defined as a positive continuous function in $\Omega$.

Moreover, using (4.35) of Lemma 4.7, we note that the infinite series in (4.37) has partial sums that constitute a Cauchy sequence in the complete metric space $L_{2}(\Omega)$. Therefore (compare [13, Chapter 3, Section 3.10])

$$
F^{*} \in L_{2}(\Omega) \text { and }\left\|F^{*}\right\|_{L_{2}(\Omega)} \leq \exp (1)\|F(\cdot, 0)\|_{L_{2}(\Omega)} .
$$

Thus by the closedness of $\stackrel{\Delta}{L}_{2}(\Omega)$ (see the corresponding statement in Remark 1.5)

$$
F^{*} \in \stackrel{\Delta}{L}_{2}(\Omega)
$$

as required by the theorem.

We wish to examine the boundary values of $F^{*}$, defined in various ways below, to obtain the boundary function $f^{*}: \partial \Omega \rightarrow \mathbb{R}$. Just as in (4.31) we define, for each $r \in(0,1)$ and then, using the coordinates $(\theta, \varphi) \in S^{2}=\partial \Omega$,

$$
f_{r}^{*}(\theta, \varphi):=F^{*}(r, \theta, \varphi) \text { for all }(\theta, \varphi) \in S^{2} .
$$

Of course, along the rays $(\theta, \varphi)$ corresponding to the countable set of points $P_{k} \in \partial \Omega$ for $k \in \mathbb{N}_{0}$, there is no finite radial limit. Accordingly we shall consider only a weak definition of

$$
f^{*}:=\lim _{r \nearrow 1} f_{r}^{*}
$$

where we verify the appropriate convergence in $L_{1}\left(S^{2}\right)$.

In order to establish the existence of the limit indicated in (4.42), and hence the boundary function $f^{*}$ of $F^{*}$, we consider the functions, for each $k \in \mathbb{N}_{0}$,

$$
f_{r}(\theta, \varphi, k):=F(r, \theta, \varphi, k) \text { for all }(\theta, \varphi) \in S^{2}
$$

and

$$
f(\cdot, \cdot, k):=\lim _{r \nearrow 1} f_{r}(\cdot, \cdot, k) \text { in } L_{1}\left(S^{2}\right),
$$

just as in (4.32) (i.e. using radial limits to define $f(\theta, \varphi, k)$ as a boundary value for $F(\cdot, \theta, \varphi, k)$ as is done for the case $k=0$ in Example 4.5).

Lemma 4.10. By the spherical symmetry of $S^{2}=\partial \Omega$, as in the earlier Lemma 4.7. we find that for $k \in \mathbb{N}_{0}$ and $r \in(0,1)$,

$$
\begin{gathered}
\left\|f_{r}(\cdot, \cdot, k)\right\|_{L_{1}\left(S^{2}\right)}=\left\|f_{r}(\cdot, \cdot, 0)\right\|_{L_{1}\left(S^{2}\right)}, \\
\|f(\cdot, \cdot, k)\|_{L_{1}\left(S^{2}\right)}=\|f(\cdot, \cdot, 0)\|_{L_{1}\left(S^{2}\right)}
\end{gathered}
$$


and

$$
\left\|f(\cdot, \cdot, k)-f_{r}(\cdot, \cdot, k)\right\|_{L_{1}\left(S^{2}\right)}=\left\|f(\cdot, \cdot, 0)-f_{r}(\cdot, \cdot, 0)\right\|_{L_{1}\left(S^{2}\right)} .
$$

Theorem 4.11. Considering all limits within the Banach space $L_{1}\left(S^{2}\right)$, we verify that

$$
\lim _{r \nearrow 1} f_{r}^{*}(\cdot, \cdot)=\sum_{k=0}^{\infty} \frac{1}{k !} f(\cdot, \cdot, k) .
$$

The boundary function $f^{*}$ of $F^{*}$, defined in (4.42) as

$$
f^{*}:=\lim _{r \nearrow 1} f_{r}^{*} \in L_{1}\left(S^{2}\right),
$$

can also be written in the form

$$
f^{*}=\sum_{k=0}^{\infty} \frac{1}{k !} f(\cdot, \cdot, k) .
$$

However $f^{*} \notin L_{2}\left(S^{2}\right)$; in fact, for each non-empty open subset $\mathcal{O} \subset S^{2}$ we observe that $\left.f^{*}\right|_{\mathcal{O}} \notin L_{2}(\mathcal{O})$.

Proof. As in Example 4.5, $f(\cdot, \cdot, k) \in L_{1}\left(S^{2}\right)$ for each $k \in \mathbb{N}_{0}$. By Lemma 4.10.

$$
\sum_{k=0}^{\infty} \frac{1}{k !} f(\cdot, \cdot, k) \text { converges in } L_{1}\left(S^{2}\right) \text {. }
$$

Next establish 4.48); for each $r<1$, again referring to 4.47),

$$
\begin{aligned}
\left\|f_{r}^{*}(\cdot, \cdot)-\sum_{k=0}^{\infty} \frac{1}{k !} f(\cdot, \cdot, k)\right\|_{L_{1}\left(S^{2}\right)} & =\left\|F^{*}(r, \cdot, \cdot)-\sum_{k=0}^{\infty} \frac{1}{k !} f(\cdot, \cdot, k)\right\|_{L_{1}\left(S^{2}\right)} \\
& =\left\|\sum_{k=0}^{\infty} \frac{1}{k !} f_{r}(\cdot, \cdot, k)-\sum_{k=0}^{\infty} \frac{1}{k !} f(\cdot, \cdot, k)\right\|_{L_{1}\left(S^{2}\right)} \\
& \leq \sum_{k=0}^{\infty} \frac{1}{k !}\left\|f_{r}(\cdot, \cdot, k)-f(\cdot, \cdot, k)\right\|_{L_{1}\left(S^{2}\right)} \\
& =\exp (1)\left\|f_{r}(\cdot, \cdot, 0)-f(\cdot, \cdot, 0)\right\|_{L_{1}\left(S^{2}\right)} ;
\end{aligned}
$$

by (4.44) we obtain the conclusion (4.48).

Finally consider the function of (4.49)

$$
f^{*}=\lim _{r \nearrow 1} f_{r}^{*} \in L_{1}\left(S^{2}\right) .
$$

First note that each term of the series

$$
\sum_{k=0}^{\infty} \frac{1}{k !} f(\theta, \varphi, k)
$$

is positive (or $+\infty$ at the corresponding singularity $P_{k} \in \partial \Omega$ ). Moreover, $f(\cdot, \cdot, k) \notin$ $L_{2}\left(S^{2}\right)$ because of its behavior near the point $P_{k}$.

In more detail, let $\mathcal{O}$ be a non-empty open subset of the spherical surface $S^{2}=$ $\partial \Omega$. Take a point $P_{k_{1}} \in \mathcal{O}$, and then $f\left(\cdot, \cdot, k_{1}\right) \notin L_{2}(\mathcal{O})$, which proves that

$$
\sum_{k=0}^{\infty} \frac{1}{k !} f(\cdot, \cdot, k) \notin L_{2}(\mathcal{O}) \text {. }
$$




\section{ACKNOWLEDGEMENTS}

The authors are indebted to

Professor W.K. Hayman, Imperial College, London,

Professor J.M. Anderson, University College, London,

for expert help and advice concerning the complex function theory of Example 4.2 and the content of Remarks 4.3 and 4.4

\section{REFERENCES}

[1] R.A. Adams. Sobolov spaces (Academic Press, London and Boston, Mass.: 1978). MR0450957 (56:9247)

[2] A. Alonso and B. Simon. The Birman-Krein-Vishik theory of self-adjoint extensions of semibounded operators. Operator Theory 4 (1980), 251-170. MR0595414 (81m:47038)

[3] J.M. Anderson and L.D. Pitt. On recurrence properties of certain lacunary series. I Crelle Jour. 377 (1987), 65-82. MR0887400 (88i:30005a)

[4] J.M. Anderson. Personal communication: April 2002.

[5] S. Bergman. The kernel function and conformal mapping. Mathematical Surveys and Monographs, 5 (American Mathematical Society, RI, USA: revised edition, 1970). MR0507701 $(58: 22502)$

[6] N. Dunford and J. Schwartz. Linear operators: I and II (Wiley, New York, USA: 1963). MR 0117523(22:8302) MR0188745(32:6181)

[7] W.N. Everitt and L. Markus. Boundary value problems and symplectic algebra for ordinary and quasi-differential operators. Mathematical Surveys and Monographs, 61 (American Mathematical Society, RI, USA:1999). MR1647856 (2000c:34030)

[8] W.N. Everitt and L. Markus. Elliptic partial differential operators and symplectic algebra. Memoirs of the American Mathematical Society 162 (2003), no. 770. MR1955204 (2004d:47054)

[9] G. Fichera. Linear elliptic differential systems and eigenvalue problems. Lecture Notes in Mathematics, 8 (Springer-Verlag, Heidelberg: 1965). MR0209639 (35:536)

[10] A. Friedman. Partial differential equations (Holt, Rinehart and Winston, New York: 1969). MR 0445088 (56:3433)

[11] G.H. Hardy. Divergent series (Oxford University Press, UK: 1949). MR0030620 (11:25a)

[12] Y. Katznelson. An introduction to harmonic analysis (Wiley, New York, USA: 1968). MR0248482(40:1734)

[13] W. Rudin. Real and complex analysis (McGraw-Hill, Inc., New York:1986). MR0924157 (88k:00002)

[14] S. Timoshenko. Theory of plates and shells (McGraw-Hill, Inc., New York:1959).

[15] J. Wloka. Partial differential equations (Cambridge University Press, UK: 1987). MR0895589 (88d:35004)

[16] K. Yosida. Functional analysis (Springer-Verlag, Heidelberg, Germany: second printing 1966). MR0180824(31:5054)

[17] A. Zygmund. Trigonometric series. I (Cambridge Press, UK: second edition 1968). MR0236587(38:4882)

School of Mathematics and Statistics, University of Birmingham, Edgbaston, BirmINGHAM B15 2TT, ENGLAND, United Kingdom

E-mail address: w.n.everitt@bham.ac.uk

School of Mathematics, Universty of Minnesota, Minneapolis, Minnesota 55455-0487

E-mail address: markus@math.umn.edu

Mathematisches Institut I, Universität Karlsruhe, D-76128 Karlsruhe, Germany

E-mail address: michael.plum@math.uni-karlsruhe.de 